University of New Orleans

ScholarWorks@UNO

$5-27-2011$

\title{
Principal angles and principal azimuths of frustrated total internal reflection and optical tunneling by an embedded low-index thin film
}

\author{
R. M.A. Azzam \\ University of New Orleans, razzam@uno.edu \\ F. F. Sudradjat
}

Follow this and additional works at: https://scholarworks.uno.edu/ee_facpubs

Part of the Electrical and Electronics Commons, and the Optics Commons

\section{Recommended Citation}

R. M. A. Azzam and F. F. Sudradjat, "Principal angles and principal azimuths of frustrated total internal reflection and optical tunneling by an embedded low-index thin film," J. Opt. Soc. Am. A 28, 1256-1261 (2011

This Article is brought to you for free and open access by the Department of Electrical Engineering at ScholarWorks@UNO. It has been accepted for inclusion in Electrical Engineering Faculty Publications by an authorized administrator of ScholarWorks@UNO. For more information, please contact scholarworks@uno.edu. 


\title{
Principal angles and principal azimuths of frustrated total internal reflection and optical tunneling by an embedded low-index thin film
}

\author{
R. M. A. Azzam ${ }^{1, *}$ and F. F. Sudradjat ${ }^{2,3}$ \\ ${ }^{1}$ Department of Electrical Engineering, University of New Orleans, New Orleans, Louisiana 70148, USA \\ ${ }^{2}$ Department of Electrical and Computer Engineering, Boston University, Boston, Massachusetts 02215, USA \\ sffsudrad@bu.edu \\ *Corresponding author: razzam@uno.edu
}

Received March 16, 2011; accepted April 13, 2011;

posted April 22, 2011 (Doc. ID 144296); published May 27, 2011

\begin{abstract}
The condition for obtaining a differential (or ellipsometric) quarter-wave retardation when $p$ - and $s$-polarized light of wavelength $\lambda$ experience frustrated total internal reflection (FTIR) and optical tunneling at angles of incidence $\phi \geq$ the critical angle by a transparent thin film (medium 1) of low refractive index $n_{1}$ and uniform thickness $d$, which is embedded in a transparent bulk medium 0 of high refractive index $n_{0}$ takes the simple form: $-\tanh ^{2} x=\tan \delta_{p} \tan \delta_{s}$, in which $x=2 \pi n_{1}(d / \lambda)\left(N^{2} \sin ^{2} \phi-1\right)^{1 / 2}, N=n_{0} / n_{1}$, and $\delta_{p}, \delta_{s}$ are 01 interface Fresnel reflection phase shifts for the $p$ and $s$ polarizations. From this condition, the ranges of the principal angle and normalized film thickness $d / \lambda$ are obtained explicitly. At a given principal angle, the associated principal azimuths $\psi_{r}$, $\psi_{t}$ in reflection and transmission are determined by $\tan ^{2} \psi_{r}=-\sin 2 \delta_{s} / \sin 2 \delta_{p}$ and $\tan ^{2} \psi_{t}=-\tan \delta_{p} / \tan \delta_{s}$, respectively. At a unique principal angle $\phi_{e}$ given by $\sin ^{2} \phi_{e}=2 /\left(N^{2}+1\right), \psi_{r}=\psi_{t}=45^{\circ}$ and linear-to-circular polarization conversion is achieved upon FTIR and optical tunneling simultaneously. The intensity transmittances of $p$ and $s$-polarized light at any principal angle are given by $\tau_{p}=\tan \delta_{p} / \tan \left(\delta_{p}-\delta_{s}\right)$ and $\tau_{s}=-\tan \delta_{s} /$ $\tan \left(\delta_{p}-\delta_{s}\right)$, respectively. The efficiency of linear-to-circular polarization conversion in optical tunneling is maximum at $\phi_{e}$. (C) 2011 Optical Society of America
\end{abstract}

OCIS codes: $\quad 240.0310,240.7040,260.2130,260.5430,260.6970,310.6860$.

\section{INTRODUCTION}

At a dielectric-conductor planar interface, a principal angle is defined as an angle of incidence at which incident linearly polarized monochromatic light of the proper azimuth, called the principal azimuth, is reflected circularly polarized [1-3]. Depending on the value of the relative complex dielectric function of the two media, one, two, or three principal angle-principal azimuth pairs may exist [2,3]. For light reflection by a transparent thin film on an absorbing substrate, there is a continuum of principal angles and an associated range of principal azimuths [ㅁ] .

A previous paper [5] presented a detailed analysis of the phase shifts that monochromatic $p$ - and $s$-polarized light of wavelength $\lambda$ experience in frustrated total internal reflection (FTIR) and optical tunneling at angles of incidence $\phi \geq$ the critical angle by a thin film of low refractive index $n_{1}$ and uniform thickness $d$, which is embedded in a transparent bulk medium of high refractive index $n_{0}$ (typically a uniform air gap, $n_{1}=1$, between parallel plane faces of two transparent prisms; Fig. 1). In a related paper []], Azzam and Spinu described an FTIR, 2-12 $\mu \mathrm{m}$ wavelength-tunable circular-polarization beam splitter that uses a variable-thickness air gap between two Ge prisms.

In this paper, closed-form solutions for the principal angles, principal azimuths, and film thicknesses that produce linear-tocircular polarization conversion in FTIR and optical tunneling by an embedded low-index thin film are obtained. The principal angle condition is considered analytically and graphically in Section 2. At a given principal angle $\phi$ and given refractive index ratio $N=n_{0} / n_{1}$, an explicit solution for the normalized film thickness $d / \lambda$ that produces dual quarter-wave retardation (QWR) in reflection and transmission is derived in Section 3. In Section $\underline{4}$, the associated principal azimuths of reflection and transmission are obtained in terms of the 01 interface Fresnel reflection phase shifts for the $p$ and $s$ polarizations. Section 5 is devoted to the special case of dual circular polarization in reflection and transmission at the unique incidence angle of equal tunneling of the $p$ and $s$ polarizations. In Section $\underline{6}$, the intensity reflectances and transmittances of $p$ - and $s$-polarized light in FTIR and optical tunneling are obtained as functions of the principal angle $\phi$ and refractive index ratio $N$. Section 7 gives a brief summary of the paper. In Appendix A, alternate expressions of the principal azimuths of reflection and transmission are presented, and, in Appendix $\underline{B}$, the condition of maximum linear-to-circular polarization conversion in optical tunneling is considered.

\section{PRINCIPAL ANGLES OF FTIR AND OPTICAL TUNNELING}

The changes of polarization that accompany FTIR and optical tunneling are determined by the ratios of complex-amplitude reflection $(R)$ and transmission $(T)$ coefficients of $p$ - and $s$-polarized light that account for coherent multiple-plane-wave interference within the embedded layer. These ellipsometric functions [7] are expressed as

$$
\begin{gathered}
\rho_{r}=R_{p} / R_{s}=\tan \psi_{r} \exp \left(j \Delta_{r}\right), \\
\rho_{t}=T_{p} / T_{s}=\tan \psi_{t} \exp \left(j \Delta_{t}\right) .
\end{gathered}
$$




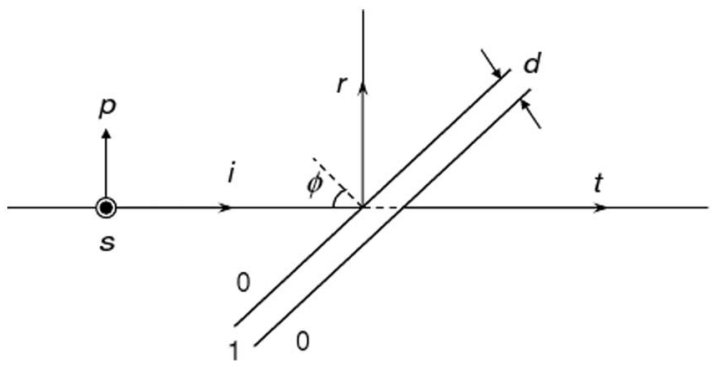

Fig. 1. Reflection and transmission of $p$ - and $s$-polarized light at an angle of incidence $\phi$ by a uniform layer of thickness $d$ and refractive index $n_{1}$ (medium 1), which is embedded in a bulk medium 0 of refractive index $n_{0}$.

In [5], it is shown that

$$
\begin{gathered}
\Delta_{r}=\Delta_{t}, \\
\tan \psi_{r} / \tan \psi_{t}=\sin \delta_{s} / \sin \delta_{p}=\left(N^{2}+1\right) \sin ^{2} \phi-1 .
\end{gathered}
$$

In Eq. (3), $N=n_{0} / n_{1}$ is the high-to-low index ratio and $\delta_{p}, \delta_{s}$ are 01 interface Fresnel reflection phase shifts for the $p$ and $s$ polarizations. Starting from results already obtained in [ㄷ], $\rho_{r}$ is written as

$$
\begin{gathered}
\rho_{r}=\frac{\tanh x \cos \delta_{s}-j \sin \delta_{s}}{\tanh x \cos \delta_{p}-j \sin \delta_{p}} \\
x=2 \pi n_{1}(d / \lambda)\left(N^{2} \sin ^{2} \phi-1\right)^{1 / 2} .
\end{gathered}
$$

At a principal angle,

$$
\operatorname{Re} \rho_{r}=0
$$

and substitution of $\rho_{r}$ from Eq. (ㅁ) in Eq. (무) leads to

$$
\begin{gathered}
\tanh ^{2} x \cos \delta_{p} \cos \delta_{s}+\sin \delta_{p} \sin \delta_{s}=0, \\
-\tanh ^{2} x=\tan \delta_{p} \tan \delta_{s} .
\end{gathered}
$$

Equation (8) is the simplest possible form of the principalangle condition in FTIR by an embedded low-index thin film. (This result is also obtained by setting the denominator of the right-hand side of Eq. (13) in [5] equal to zero.) For specified values of $n_{1}, N$, Eq. ( $\underline{8}$ ) represents the constraint on $d / \lambda, \phi$ such that the overall differential reflection and transmission phase shifts are quarter-wave, i.e., $\Delta_{r}=\Delta_{t}=\pi / 2$.

From the known expressions of the 01 interface Fresnel reflection phase shifts $\delta_{\nu}(\nu=p, s)$ []ㅡ, the right-hand side of Eq. (8) can be cast as a function of $\phi$ of the form

$$
\tan \delta_{p} \tan \delta_{s}=\left(\frac{\sin ^{2} \phi_{p}}{\sin ^{2} \phi_{s}}\right) \frac{\left(\sin ^{2} \phi-\sin ^{2} \phi_{c}\right)\left(1-\sin ^{2} \phi\right)}{\left(\sin ^{2} \phi-\sin ^{2} \phi_{p}\right)\left(\sin ^{2} \phi-\sin ^{2} \phi_{s}\right)} .
$$

In Eq. (9), $\phi_{c}=\sin ^{-1}(1 / N)$ is the critical angle and $\phi_{p}, \phi_{s}$ are the angles of incidence at which $\delta_{p}=\pi / 2$ and $\delta_{s}=\pi / 2$, respectively [8].

As an example, consider a uniform air gap $\left(n_{1}=1\right)$ between two Ge prisms $(N=4)$ in the IR. Figure 2 shows the product of tangents given by Eq. (9) as a function of $\phi$ for $\phi_{c} \leq$ $\phi \leq 90^{\circ}$ as a continuous line. Singularities of this function appear at $\phi_{p}=14.90^{\circ}$ and $\phi_{s}=46.79^{\circ}$ as expected. In Fig. 2 , a family of curves (dashed curves) that represent the left-hand side of Eq. (8) is also plotted versus $\phi$ for discrete values of $d / \lambda=0.005,0.02$ to 0.20 in equal steps of 0.02 , and 10 . Solutions of Eq. ( $\underline{8}$ ) correspond to points of intersection of the negative branch of the product-of-tangents function $\left(\phi_{p}<\phi<\phi_{s}\right)$ and the dashed lines that represent $-\tanh ^{2} x$ (e.g., points $\mathrm{A}$ and $\mathrm{B}$ for $d / \lambda=0.08$ ). Principal angles of the Ge-air-Ge system cease to exist for very thin films with $d / \lambda<0.059$. At large film thicknesses (e.g., $d / \lambda \geq 10$ ), optical tunneling is negligible and TIR at the 01 interface is restored. In this large-thickness limit, $\tanh ^{2} x=1$ and Eq. (8) reduces to

$$
\tan \delta_{p} \tan \delta_{s}=-1 \text {. }
$$

Equation ( $\underline{10}$ ) indicates that $\delta_{p}-\delta_{s}=\pi / 2$, which is the principal-angle condition of TIR at the 01 interface. The full range of principal angles $\phi_{1} \leq \phi \leq \phi_{2}$ is defined in Fig. 2 by the points of intersection $P_{1}$ and $P_{2}$ at which Eq. (10) is satisfied. The corresponding limiting angles $\phi_{1}$ and $\phi_{2}$ are given by [8]

$$
\sin ^{2} \phi_{1,2}=\left[\left(N^{2}+1\right) \mp\left(N^{4}-6 N^{2}+1\right)^{1 / 2}\right] / 4 N^{2} .
$$

Acceptable solutions of Eq. (11) exist if

$$
N \geq \sqrt{2}+1=2.414 .
$$

For the Ge-air interface $N=4, \phi_{1}=15.04^{\circ}$ (which is slightly above the critical angle $\phi_{c}=14.48^{\circ}$ ) and $\phi_{2}=42.93^{\circ}$.

\section{FILM THICKNESS FOR QWR AT A GIVEN PRINCIPAL ANGLE}

For an index ratio $N>2.414$ and a principal angle $\phi$ in the range $\phi_{1} \leq \phi \leq \phi_{2}$ defined by Eq. (11), the value of $\tanh x$ that satisfies the principal-angle condition is determined by Eqs. (8) and (9). From $\tanh x$,

$$
X=\exp (-2 x)=(1-\tanh x) /(1+\tanh x)
$$

is calculated. Next, the normalized film thickness that produces QWR in reflection and transmission $\left(\Delta_{r}=\Delta_{t}=\pi / 2\right)$ is obtained from Eqs. (ㅁ) and (13) as

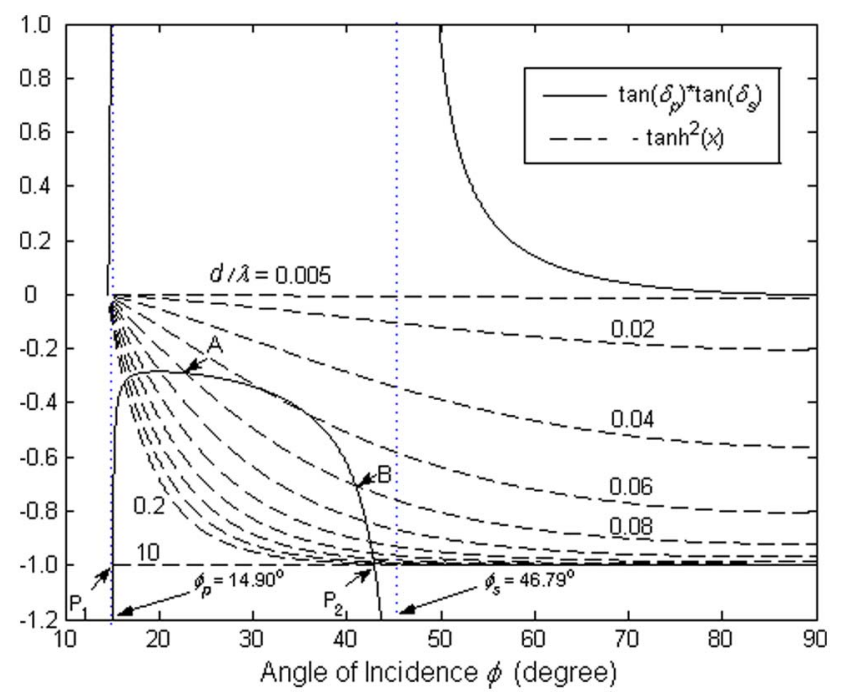

Fig. 2. (Color online) Graphical construction that illustrates the range of possible solutions of Eq. (8)). 


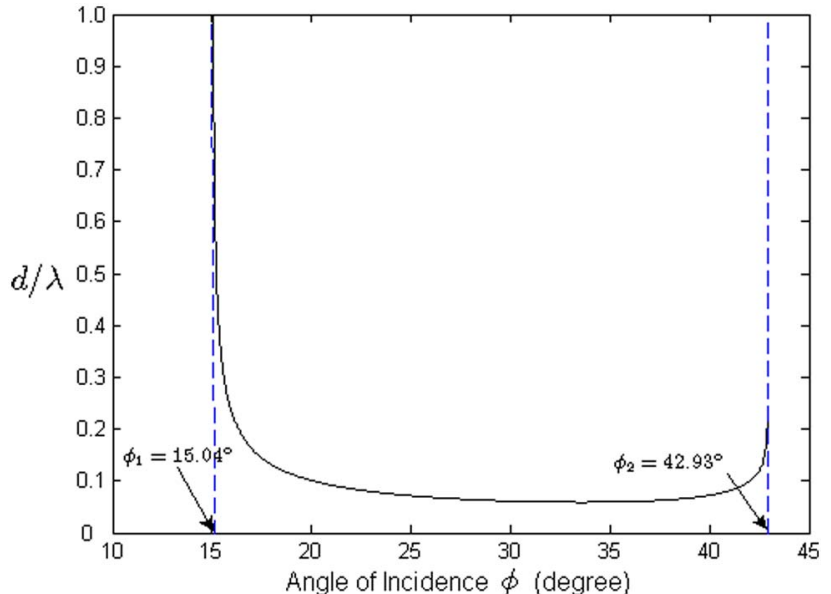

Fig. 3. (Color online) Normalized thickness $d / \lambda$ of a uniform air gap between two IR-transparent Ge prisms $\left(n_{1}=1, N=4\right)$ that produces $\Delta_{r}=\Delta_{t}=\pi / 2$ is plotted as a function of principal angle $\phi$ over the full range from $\phi_{1}=15.04^{\circ}$ to $\phi_{2}=42.93^{\circ}$.

$$
d / \lambda=-(\ln X) /\left[4 \pi n_{1}\left(N^{2} \sin ^{2} \phi-1\right)^{1 / 2}\right] .
$$

As an example, the above algorithm is applied to a uniform air gap between two IR-transparent Ge prisms $\left(n_{1}=1, N=4\right)$ at $\phi=30^{\circ}$; this gives $\delta_{p}=165.75^{\circ}, \delta_{s}=53.13^{\circ}, \tanh x=$ $\left(-\tan \delta_{p} \tan \delta_{s}\right)^{1 / 2}=0.581914, \quad X=0.264291, \quad$ and $\quad d / \lambda=$ 0.061138 .

Use of the same algorithm for the Ge-air-Ge system over the full range of principal angles from $\phi_{1}=15.04^{\circ}$ to $\phi_{2}=$ $42.93^{\circ}, d / \lambda$ at which $\Delta_{r}=\Delta_{t}=\pi / 2$ is obtained as a function of principal angle $\phi$, as shown in Fig. 3 . Except for a change of scale of the ordinate axis, this graph is the same as the one obtained by Azzam and Spinu (Fig. 2 in [6]) using an iterative numerical technique.

\section{REFLECTION AND TRANSMISSION PRINCIPAL AZIMUTHS AT A GIVEN PRINCIPAL ANGLE}

At a principal angle, $\cos \Delta_{r}=0, \sin \Delta_{r}=1, \operatorname{Re} \rho_{r}=0$ [Eq. ( $\left.\underline{6}\right)$ ], and $\rho_{r}$ reduce to

$$
\rho_{r}=j \tan \psi_{r}
$$

In Eq. (15), $\psi_{r}$ is the associated principal azimuth (the angle between the electric-field vector of incident linearly polarized light and the plane of incidence) that produces circularly polarized reflected light. From Eqs. (4) and (15), we obtain

$$
\operatorname{Im} \rho_{r}=\tan \psi_{r}=\frac{\tanh ^{2} x \sin \left(\delta_{p}-\delta_{s}\right)}{\tanh ^{2} x \cos ^{2} \delta_{p}+\sin ^{2} \delta_{p}} .
$$

Substitution of $\tanh ^{2} x=-\tan \delta_{p} \tan \delta_{s}$ [Eq. (ㅁ)] in Eq. (16) and use of trigonometric identities lead to an explicit expression for the principal azimuth $\psi_{r}$ in terms of the 01 interface TIR phase shifts $\delta_{\nu}(\nu=p, s)$ :

$$
\tan ^{2} \psi_{r}=-\sin 2 \delta_{s} / \sin 2 \delta_{p}
$$

At the same principal angle, the principal azimuth $\psi_{t}$ that produces circular polarization of the transmitted (instead of reflected) light is derived from Eqs. (3) and (17) as

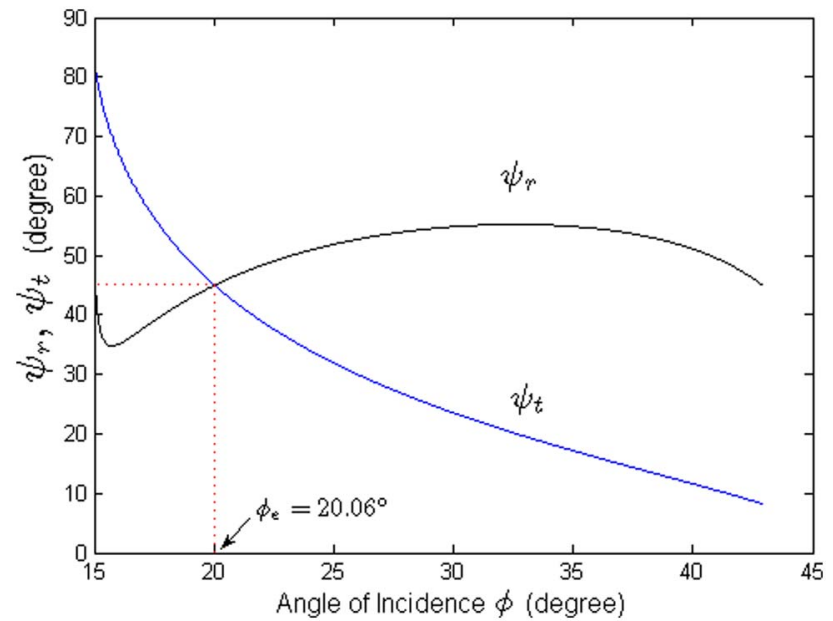

Fig. 4. (Color online) Reflection and transmission principal azimuths $\psi_{r}, \psi_{t}$ [Eqs. (17) and (18)] are plotted as functions of the principal angle $\phi$ for a uniform air gap between two IR-transparent Ge prisms $\left(n_{1}=1, N=4\right)$ over the full range of principle angles $\phi_{1} \leq \phi \leq \phi_{2}$.

$$
\tan ^{2} \psi_{t}=-\tan \delta_{p} / \tan \delta_{s}
$$

An air gap between two Ge prisms $\left(n_{1}=1, N=4\right)$ with thickness $d / \lambda=0.061138$ has a principal angle $\phi=30^{\circ}$ (Section 3). Substitution of $\delta_{p}=165.75^{\circ}$ at $\delta_{s}=53.13^{\circ}, \phi=$ $30^{\circ}$ in Eqs. (17) and (18) gives $\psi_{r}=54.816^{\circ}, \psi_{t}=23.578^{\circ}$. Calculation of $\psi_{r}, \psi_{t}$ as functions of $\phi$ over the full range of principle angles $\phi_{1} \leq \phi \leq \phi_{2}$ of the Ge-air-Ge system produces the two curves shown in Fig. 4 .

In Appendix $\underline{\mathrm{A}}$, we give alternate explicit expressions of $\psi_{r}$, $\psi_{t}$ as functions of $\phi$ for a given $N$ and locate the angular positions of the minimum and maximum of the $\psi_{r}$-versus- $\phi$ curve.

\section{DUAL CIRCULAR POLARIZATION OF REFLECTED AND TRANSMITTED LIGHT AT ONE PRINCIPAL ANGLE}

From Eq. (3), the principal azimuths of FTIR and optical tunneling are equal, $\psi_{r}=\psi_{t}$, at one principal angle $\phi_{e}$, given by

$$
\sin ^{2} \phi_{e}=2 /\left(N^{2}+1\right) .
$$

$\phi_{e}$ of Eq. (19) is the incidence angle of equal tunneling of $p$ - and $s$-polarized light $\left[\underline{9,10]}\right.$ so that $\psi_{r}=\psi_{t}=45^{\circ}$. In Fig. 4, for $N=4, \psi_{r}=\psi_{t}=45^{\circ}$ at $\phi_{e}=\sin ^{-1}(2 / 17)^{1 / 2}=20.06^{\circ}$.

It is worthwhile to recall that $\phi_{e}$ is the angle of incidence at which phase difference $\left(\delta_{p}-\delta_{s}\right)$ is maximum and the average phase shift is $\left(\delta_{p}+\delta_{s}\right) / 2=\pi / 2$ [8].

Also, at $\phi_{e}$, the Fresnel reflection phase shifts at the 01 interface [] simplify to

$$
\tan \left(\delta_{s} / 2\right)=1 / N, \quad \tan \left(\delta_{p} / 2\right)=N .
$$

Equation (20) indicates that $\delta_{s}$ and $\delta_{p}$ at $\phi_{e}$ are equal to double the Brewster angles of internal and external reflection at the 01 interface, respectively.

Still another curious property of $\phi_{e}$ is that, for a given $N$, the product of tangents given by Eq. (9) reaches a maximum at that angle. This can be proved by substituting $\sin ^{2} \phi=u$ in Eq. (9) and setting the derivative of the right-hand side with respect to $u$ equal to zero. By use of Eq. (20) and the trigonometric identity $\tan x=2 \tan (x / 2) /\left[1-\tan ^{2}(x / 2)\right]$, the maximum value of $\tan \delta_{p} \tan \delta_{s}$ at $\phi_{e}$ is obtained: 


$$
\left(\tan \delta_{p} \tan \delta_{s}\right)_{\max }=-4 N^{2} /\left(N^{2}-1\right)^{2} .
$$

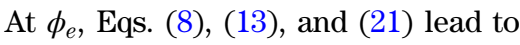

$$
\begin{gathered}
\tanh x=2 N /\left(N^{2}-1\right), \\
X=\left(N^{2}-2 N-1\right) /\left(N^{2}+2 N-1\right) .
\end{gathered}
$$

The value of $d / \lambda$ that produces dual QWR with equal throughput for the $p$ and $s$ polarizations in FTIR and optical tunneling at $\phi_{e}$ is obtained by substituting $X$ from Eq. (23) and $\left(N^{2} \sin ^{2} \phi_{e}-1\right)^{1 / 2}=\left[\left(N^{2}-1\right) /\left(N^{2}+1\right)\right]^{1 / 2}$ in Eq. (14); this gives

$$
d / \lambda=\left(4 \pi n_{1}\right)^{-1}\left(\frac{N^{2}+1}{N^{2}-1}\right)^{1 / 2} \ln \left(\frac{N^{2}+2 N-1}{N^{2}-2 N-1}\right) .
$$

For the Ge-air-Ge system $\left(n_{1}=1, N=4\right), \phi_{e}=20.06^{\circ}$ and the normalized air-gap thickness for dual QWR in reflection and transmission is obtained from Eq. (24):

$$
d / \lambda=(4 \pi)^{-1}(17 / 15)^{1 / 2} \ln (23 / 7)=0.100778
$$

The closed-form solution for $d / \lambda$ [Eq. (24)] supersedes any iterative numerical approach [6] . Figure $\underline{5}$ is a graph of $d / \lambda$ as a function of $N$ calculated form Eq. (24) over the range $2.5 \leq N \leq 6.0$

\section{REFLECTANCES AND TRANSMITTANCES OF $p$ - AND $s$ - POLARIZED LIGHT AT A GIVEN PRINCIPAL ANGLE}

The intensity reflectances and transmittances of $p$ - and $s$-polarized light are obtained by taking the squared absolute value of the corresponding complex-amplitude reflection and transmission coefficients $R_{p}, R_{s} ; T_{p}, T_{s}$ given in [5]. The resulting expressions are functions of the film thickness parameter $x$ [Eq. (5)] and the 01 interface Fresnel reflection phase shifts $\delta_{\nu}(\nu=p, s)$ :

$$
\begin{array}{cc}
\mathrm{R}_{\nu}=\left|R_{\nu}\right|^{2}=\frac{\cosh 2 x-1}{\cosh 2 x-\cos 2 \delta_{\nu}}, & \nu=p, s ; \\
\tau_{\nu}=1-\left|R_{\nu}\right|^{2}=\frac{1-\cos 2 \delta_{\nu}}{\cosh 2 x-\cos 2 \delta_{\nu}}, & \nu=p, s .
\end{array}
$$

From Eq. (27), the principal-angle condition of Eq. (8), and the identity

$$
\cosh 2 x=\left(1+\tanh ^{2} x\right) /\left(1-\tanh ^{2} x\right),
$$

the following expressions of the $p$ and $s$ intensity transmittances are obtained:

$$
\tau_{p}=\tan \delta_{p} / \tan \left(\delta_{p}-\delta_{s}\right), \quad \tau_{s}=-\tan \delta_{s} / \tan \left(\delta_{p}-\delta_{s}\right)
$$

Note that, from Eq. (29), $\tau_{p} / \tau_{s}=\tan ^{2} \psi_{t}=-\tan \delta_{p} / \tan \delta_{s}$ in agreement with Eq. (18). The corresponding intensity reflectances are given by

$$
\mathrm{R}_{\nu}=1-\tau_{\nu} \quad \nu=p, s
$$

Substitution of $\delta_{p}, \delta_{s}\left(\delta_{p}-\delta_{s}\right)$ as functions of $N$ and $\phi$ from [] in Eqs. (29) yields

$$
\begin{gathered}
\tau_{p}=\frac{2 N^{2} \sin ^{4} \phi-\left(N^{2}+1\right) \sin ^{2} \phi+1}{-\left(N^{4}+1\right) \sin ^{4} \phi+\left(N^{2}+1\right) \sin ^{2} \phi}, \\
\tau_{s}=\frac{2 N^{2} \sin ^{4} \phi-\left(N^{2}+1\right) \sin ^{2} \phi+1}{2 N^{2} \sin ^{4} \phi-\left(N^{2}+1\right) \sin ^{2} \phi}
\end{gathered}
$$

Equations (31) and (32) are valid over the full range of principal angles $\phi_{1} \leq \phi \leq \phi_{2}$ defined by Eq. (1ㅡ) and give exact mathematical representation of the families of curves presented in Fig. 5 in [6]. Both transmittances are zero at the limiting angles $\phi_{1}$ and $\phi_{2}$ given by Eq. (11) as can be verified by setting the common numerator of Eqs. (31) and (32) equal to zero.

For a given $N$, the transmittance $\tau_{p}(\phi)$ of Eq. (31) reaches a maximum at a principal angle of incidence given by

$$
\sin ^{2} \phi_{p \max }=\left(N^{2}+1\right) /\left(N^{2}-1\right)^{2} .
$$

Likewise, for a given $N$, the transmittance $\tau_{s}(\phi)$ of Eq. (32) reaches a maximum at a principal angle of incidence given by

$$
\sin ^{2} \phi_{s \max }=\left(N^{2}+1\right) / 4 N^{2} .
$$

Equations (33) and (34) are obtained by substituting $\sin ^{2} \phi=u$ in Eqs. (31) and (32) and setting the derivative of the righthand side of each equation with respect to $u$ equal to zero.

For a given $N$, the maximum transmittances at $\phi_{p \text { max }}$ and $\phi_{s \max }$ are equal,

$$
\tau_{p \max }=\tau_{s \max }=\frac{N^{4}-6 N^{2}+1}{N^{4}+2 N^{2}+1},
$$

and the corresponding principal azimuths are related by

$$
\begin{gathered}
\psi_{t}\left(\phi_{s \max }\right)=90^{\circ}-\psi_{t}\left(\phi_{p \max }\right), \\
\psi_{t}\left(\phi_{p \max }\right)=\arctan \left[\frac{N^{6}-3 N^{4}-3 N^{2}+1}{2 N^{2}\left(N^{2}+1\right)}\right]^{1 / 2} .
\end{gathered}
$$

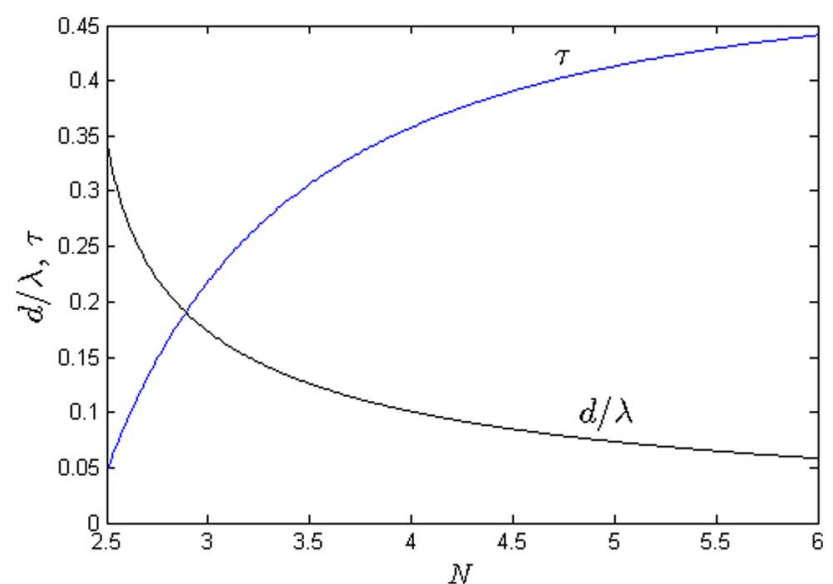

Fig. 5. (Color online) Normalized thickness $d / \lambda$ of a low-index embedded layer that produces circular polarization in FTIR and optical tunneling at $\phi_{e}$ and $\psi_{r}=\psi_{t}=45^{\circ}$ [Eq. (24)] is plotted as a function of the refractive index ratio $N$ over the range $2.5 \leq N \leq 6.0$. Equal transmittance of $p$ - and $s$-polarized light $\tau_{p}\left(\phi_{e}\right)=\tau_{s}\left(\phi_{e}\right)=\tau$ [Eq. (39)] is also shown as a function of $N$. 
For the Ge-air-Ge system in the IR, $N=4$ and Eqs. (33)-(37) give

$$
\begin{aligned}
\phi_{p \max } & =15.95^{\circ}, \quad \phi_{s \max }=31.02^{\circ} ; \\
\tau_{p \max } & =\tau_{s \max }=0.5571 ; \quad \psi_{t}\left(\phi_{p \max }\right)=67.84^{\circ}, \\
\psi_{t}\left(\phi_{s \max }\right) & =22.16^{\circ} .
\end{aligned}
$$

The analytical expressions presented here fully explain the results previously obtained by Azzam and Spinu using a numerical technique (see Fig. 5 in [6] ]).

When the denominators of the right-hand sides of Eqs. (31) and (32) are equal, the $p$ and $s$ transmittances become equal, and the condition of equal tunneling of the $p$ and $s$ polarizations [Eq. (19)] at $\phi=\phi_{e}$ is recovered. At $\phi_{e}$, the equal throughputs for the $p$ and $s$ polarizations are given by

$$
\tau_{p}\left(\phi_{e}\right)=\tau_{s}\left(\phi_{e}\right)=1-\frac{1}{2}\left(\frac{N^{2}+1}{N^{2}-1}\right)^{2} .
$$

The same transmittance of $p$ - and $s$-polarized light at $\phi=\phi_{e}$ $\left[\tau_{p}\left(\phi_{e}\right)=\tau_{s}\left(\phi_{e}\right)=\tau\right]$ is plotted as a function of $N$ in Fig. $\underline{5}$. For $N=4$, Eq. (39) gives $\tau_{p}\left(\phi_{e}\right)=\tau_{s}\left(\phi_{e}\right)=0.35778$ in agreement with [6].

Another curious result of this section is that $\phi_{s \max }$ given by Eq. (34) is also the angle of incidence at which $\delta_{p}=3 \delta_{s}$ [ㅁ] .

In Appendix B, it is shown that the efficiency of linearto-circular polarization conversion upon optical tunneling is maximum at $\phi=\phi_{e}$.

\section{CONCLUSION}

Highlights of this paper are summarized as follows.

1. The principal-angle condition of FTIR and optical tunneling by an embedded low-index thin film is given in concise form by Eq. ()ㅡ. For selected refractive indices $n_{1}, N$, Eq. ( $\left.\underline{8}\right)$ represents the constraint on the normalized film thickness and principal angle $(d / \lambda, \phi)$ such that the differential phase shifts in reflection and transmission are quarter-wave. Figure 2 illustrates the domain of $d / \lambda, \phi$ for which acceptable solutions of Eq. (8) exist when $n_{1}=1, N=4$.

2. For given values of $n_{1}, N, d / \lambda$ that leads to QWR in reflection and transmission (i.e., $\Delta_{r}=\Delta_{t}=\pi / 2$ ) at a principal angle $\phi$ is explicitly determined by Eq. (14).

3. Equations (17) and (18) determine the reflection and transmission principal azimuths $\psi_{r}, \psi_{t}$ in terms of the 01 interface Fresnel reflection phase shifts $\delta_{\nu}(\nu=p, s)$.

4. At the angle $\phi_{e}$ given by Eq. (19), $\psi_{r}=\psi_{t}=45^{\circ}$, and circular polarization in FTIR and optical tunneling is achieved simultaneously at thickness-to-wavelength ratio $d / \lambda$ given by Eq. (24).

5. At a given principal angle, the throughputs for the $p$ and $s$ polarizations in optical tunneling are given by Eqs. (29), (31), and (32). These transmittances have maxima at principal angles given by Eqs. (33) and (34), respectively.

6 . The efficiency of linear-to-circular polarization conversion upon optical tunneling is maximum [Eq. (B7)] at $\phi=\phi_{e}$.

\section{APPENDIX A}

By substituting the Fresnel interface reflection phase shifts $\delta_{\nu}$ $(\nu=p, s)$ as functions of $N, \phi$ from [8] in Eqs. (18) and (17), we obtain

$$
\begin{gathered}
\tan ^{2} \psi_{t}=-\left(\frac{u_{p}}{u_{s}}\right)\left(\frac{u-u_{s}}{u-u_{p}}\right), \\
\tan ^{2} \psi_{r}=-\left(\frac{4 u_{p}}{u_{s} u_{e}^{2}}\right)\left(\frac{\left(u-u_{s}\right)\left(u-0.5 u_{e}\right)^{1 / 2}}{\left(u-u_{p}\right)}\right) .
\end{gathered}
$$

In Eq. ( $\underline{\mathrm{A} 1})$ and (A2),$u=\sin ^{2} \phi$ and $u_{p}, u_{s}, u_{e}$ are the values of $u$ evaluated at the special angles $\phi_{p}, \phi_{s}, \phi_{e}=\phi_{a}$ defined in [8] that depend on $N$ only. Equations (A1) and (A2) provide explicit expressions for the principal azimuths $\psi_{r}, \psi_{t}$ as functions of the principal angle $\phi$ for any given $N$. For $N=4$, the curves of $\psi_{r}, \psi_{t}$ versus $\phi$ are plotted in Fig. 4 .

In Fig. 4 , it is apparent that $\psi_{t}$ decreases monotonically as $\phi$ increases, whereas $\psi_{r}$ exhibits a minimum and a maximum as a function of $\phi$. The angular positions of the minimum and maximum of $\psi_{r}$ are determined by setting the first derivative of the right-hand side of Eq. (A2) with respect to $u$ equal to zero. This gives a quadratic equation in $u$ whose roots are

$$
\begin{aligned}
u_{\mp}= & (1 / 4)\left\{\left(3 u_{p}+u_{s}\right)\right. \\
& \left.\mp \sqrt{\left(3 u_{p}+u_{s}\right)^{2}+4 u_{e}\left(u_{s}-u_{p}\right)-16 u_{p} u_{s}}\right\} .
\end{aligned}
$$

For $N=4, u_{p}=17 / 257, \quad u_{s}=17 / 32, \quad u_{e}=2 / 17$, and Eq. (A3) gives $u_{-}=0.073719, \phi_{-}=15.7542^{\circ} ; u_{+}=0.291128$, $\phi_{+}=32.6539^{\circ}$, which exactly locate the angular positions of the minimum and maximum of $\psi_{r}$ in Fig. $\underline{4}$.

\section{APPENDIX B}

At a given principal angle-principal azimuth pair $\left(\phi, \psi_{t}\right)$, incident linearly polarized light of intensity $I_{i}$ is partially transmitted as circularly polarized with intensity $I_{t}$ given by

$$
I_{t}=I_{i}\left(\tau_{p} \cos ^{2} \psi_{t}+\tau_{s} \sin ^{2} \psi_{t}\right) .
$$

The efficiency of linear-to-circular polarization conversion in optical tunneling is given by

$$
\eta_{\mathrm{LTC}}=I_{t} / I_{i}=\left(\tau_{p} \cos ^{2} \psi_{t}+\tau_{s} \sin ^{2} \psi_{t}\right) .
$$

Given that $\tau_{p} / \tau_{s}=\tan ^{2} \psi_{t}$, Eq. (트) becomes

$$
\eta_{\mathrm{LTC}}=I_{t} / I_{i}=2 \tau_{p} \cos ^{2} \psi_{t}=2 \tau_{s} \sin ^{2} \psi_{t}
$$

Substitution of Eqs. (18) and (29) and the Fresnel interface reflection phase shifts $\delta_{\nu}(\nu=\bar{p}, s)$ as functions of $N, \phi$ [ $\left.\underline{8}\right]$ in Eq. (B3) leads to

$$
\eta_{\mathrm{LTC}}=\left[u_{p} /\left(u_{p}-u_{s}\right)\right]\left[2-2 u_{s} u^{-1}+u_{c} u^{-2}\right] .
$$

In Eq. ( $\underline{\mathrm{B} 4}), u=\sin ^{2} \phi$ and $u_{c}, u_{p}, u_{s}$ are the values of $u$ evaluated at the angles $\phi_{c}, \phi_{p}, \phi_{s}$ defined in [8]. By setting

$$
d \eta_{\mathrm{LTC}} / d u=0
$$

in Eq. (B4), the value of $u$ at which $\eta_{\text {LTC }}$ is maximum is obtained:

$$
u=u_{c} / u_{s}=2 /\left(N^{2}+1\right)
$$

Equations (19) and ( $\underline{\mathrm{B} 6})$ confirm that $\eta_{\mathrm{LTC}}$ is maximum at the angle $\phi_{e}$ of equal tunneling of the $p$ and $s$ polarizations 
$\left(\psi_{t}=45^{\circ}\right)$; the associated maximum value of $\eta_{\mathrm{LTC}}$ is obtained from Eqs. (B3) and (39) as

$$
\eta_{\mathrm{LTC}}^{\max }=\tau_{p}\left(\phi_{e}\right)=\tau_{s}\left(\phi_{e}\right) 1-\frac{1}{2}\left(\frac{N^{2}+1}{N^{2}-1}\right)^{2} .
$$

\section{REFERENCES}

1. H. B. Holl, "Specular reflection and characteristics of reflected light," J. Opt. Soc. Am. 57, 683-690 (1967).

2. R. M. A. Azzam, "Contours of constant principal angle and constant principal azimuth in the complex $\varepsilon$ plane," J. Opt. Soc. Am. 71, 1523-1528 (1981).

3. R. M. A. Azzam and A. Alsamman, "Plurality of principal angles for a given pseudo-Brewster angle when polarized light is reflected at a dielectric-conductor interface," J. Opt. Soc. Am. A 25, 2858-2864 (2008).
4. R. M. A. Azzam and A.-R. M. Zaghloul,"Principal angle, principal azimuth, and principal-angle ellipsometry of film-substrate systems," J. Opt. Soc. Am. 67, 1058-1065 (1977).

5. R. M. A. Azzam, "Phase shifts in frustrated total internal reflection and optical tunneling by an embedded low-index thin film," J. Opt. Soc. Am. A 23, 960-965 (2006).

6. R. M. A. Azzam and C. L. Spinu,"Linear-to-circular polarization transformation upon optical tunneling through an embedded low-index film," Opt. Lett. 30, 3183-3185 (2005).

7. R. M. A. Azzam and N. M. Bashara, Ellipsometry and Polarized Light (North-Holland, 1987).

8. R. M. A. Azzam, "Phase shifts that accompany total internal reflection at a dielectric-dielectric interface," J. Opt. Soc. Am. A 21, 1559-1563 (2004).

9. P. W. Baumeister, "Optical tunneling and its application to optical filters," Appl. Opt. 6, 897-905 (1967).

10. R. W. Astheimer, G. Falbel, and S. Minkowitz, "Infrared modulation by means of frustrated total internal reflection," Appl. Opt. 5, 87-91 (1966). 